\title{
Visual Selection and Posterior Parietal Cortex: Effects of Repetitive Transcranial Magnetic Stimulation on Partial Report Analyzed by Bundesen's Theory of Visual Attention
}

\author{
June Hung, ${ }^{1,2,3}$ Jon Driver, ${ }^{1}$ and Vincent Walsh ${ }^{1}$ \\ ${ }^{1}$ Institute of Cognitive Neuroscience and Department of Psychology, University College London, London WC1N 3AR, United Kingdom, ${ }^{2}$ Department of \\ Experimental Psychology, University of Oxford, Oxford OX1 3UD, United Kingdom, and ²Department of Neurology, Chang Gung Memorial Hospital, Taipei \\ 10507, Taiwan
}

\begin{abstract}
Posterior parietal cortex (PPC) may contribute to visual selection by exerting top-down influences on visual processing. To seek direct evidence for this, we used $10 \mathrm{~Hz}$ repetitive transcranial magnetic stimulation (rTMS) over right or left PPC in nine healthy volunteers during a partial (selective) report task that allows quantitative assessment of top-down control and other parameters. Participants reported digits in a relevant color ("targets") but not those in an irrelevant color ("nontargets") from a brief masked display, in which a target could appear alone or together with an accompanying item (nontarget or target) in the same or opposite hemifield. Generally, a given target is identified better when presented with a nontarget than with another target, indicating top-down selection of task-relevant targets; this applied here with no rTMS or left PPC rTMS. However, rTMS over the right PPC changed the performance pattern. A left target no longer impeded report of a right target more strongly than did a left nontarget, whereas the greater impact of a right target than a right nontarget in disrupting report of a left target was increased. Formal analysis in terms of Bundesen's (1990) theory of visual attention indicated that right PPC rTMS diminished top-down control for the left hemifield while enhancing this for the right hemifield, particularly for bilateral two-item displays. These findings indicate a role for right PPC in top-down spatial selection, which applies even when the target is defined by a nonspatial property (here color).
\end{abstract}

Key words: parietal; transcranial magnetic stimulation; visual selectivity; extinction; spatial; attention

\section{Introduction}

Multiple visual stimuli may compete for limited neural resources (Broadbent, 1958; Harter and Aine, 1984; Bundesen, 1990; Desimone and Duncan, 1995; Driver and Frackowiak, 2001). In visual cortex, competition involves mutual suppression of evoked responses by multiple stimuli (Kwong et al., 1992; Kastner et al., 1998; Reynolds et al., 1999; Fink et al., 2000), but task-relevant stimuli may be advantaged in such competition by top-down signals (Desimone and Duncan, 1995; Desimone, 1998; Reynolds et al., 1999). Some top-down influences on visual cortex may originate from posterior parietal cortex (PPC) (Kastner and Ungerleider, 2000, 2001; Driver and Frackowiak, 2001), as suggested by functional neuroimaging (Hopfinger et al., 2000; Yantis et al., 2002) and by effects of brain damage in patients (Heilman and Valenstein, 1979; Mesulam, 1981; Driver and Vuilleumier, 2001).

Received March 4, 2005; revised Aug. 15, 2005; accepted Sept. 6, 2005.

The Wellcome Trust supported this research with a programme grant (J.D.), a project grant (V.W.), and an equipment grant to the Transcranial Magnetic Stimulation Laboratory at the Institute of Cognitive Neuroscience, University College London. J.H. holds a Swire scholarship from John Swire \& Sons Ltd. (London, UK). J.D. is supported by the Wellcome Trust and a Royal Society-Wolfson Research Merit Award. V.W. is supported by the Royal Society. We thank Prof. C. Bundesen and Dr. S. Kyllingsbæk for providing the program computing TVA parameters.

Correspondence should be addressed to Dr. J. Hung, Department of Neurology, Chang Gung Memorial Hospital, Taipei 10507, Taiwan. E-mail: jhung@cgmh.org.tw.

DOI:10.1523/JNEUROSCI.0879-05.2005

Copyright $\odot 2005$ Society for Neuroscience $\quad$ 0270-6474/05/259602-11\$15.00/0
However, inferences drawn solely from these methods have some limitations (Walsh and Pascual-Leone, 2003). Functional neuroimaging alone cannot establish the causal involvement of activated areas in attentional control. Patient studies can be hampered by the extent of lesions and possible reorganization after permanent damage.

To seek more direct evidence for the role of PPC in controlling visual selection, we used repetitive transcranial magnetic stimulation (rTMS), which can be used to test the causal role of particular cortical areas by assessing the disruptions that rTMS produces (for review, see Walsh and Cowey, 2000; Walsh and Pascual-Leone, 2003). We applied rTMS to right or left PPC (or none) while participants selectively reported digits in a relevant color ("targets") but not those in an irrelevant color ("nontargets") from brief displays without eye movements (i.e., "partial" or selective report, as opposed to nonselective "whole report"). This classic partial-report procedure (cf. Sperling, 1960; von Wright, 1968) allows analysis in terms of Bundesen's (1990) “theory of visual attention" (TVA) to assess top-down control of visual selection formally.

TVA can be considered a mathematical implementation of limited capacity and biased competition. In TVA, processing efficiency for a single item presented alone characterizes its sensory effectiveness. Competition between concurrent stimuli can impair their processing, revealing their attentional weights relative 

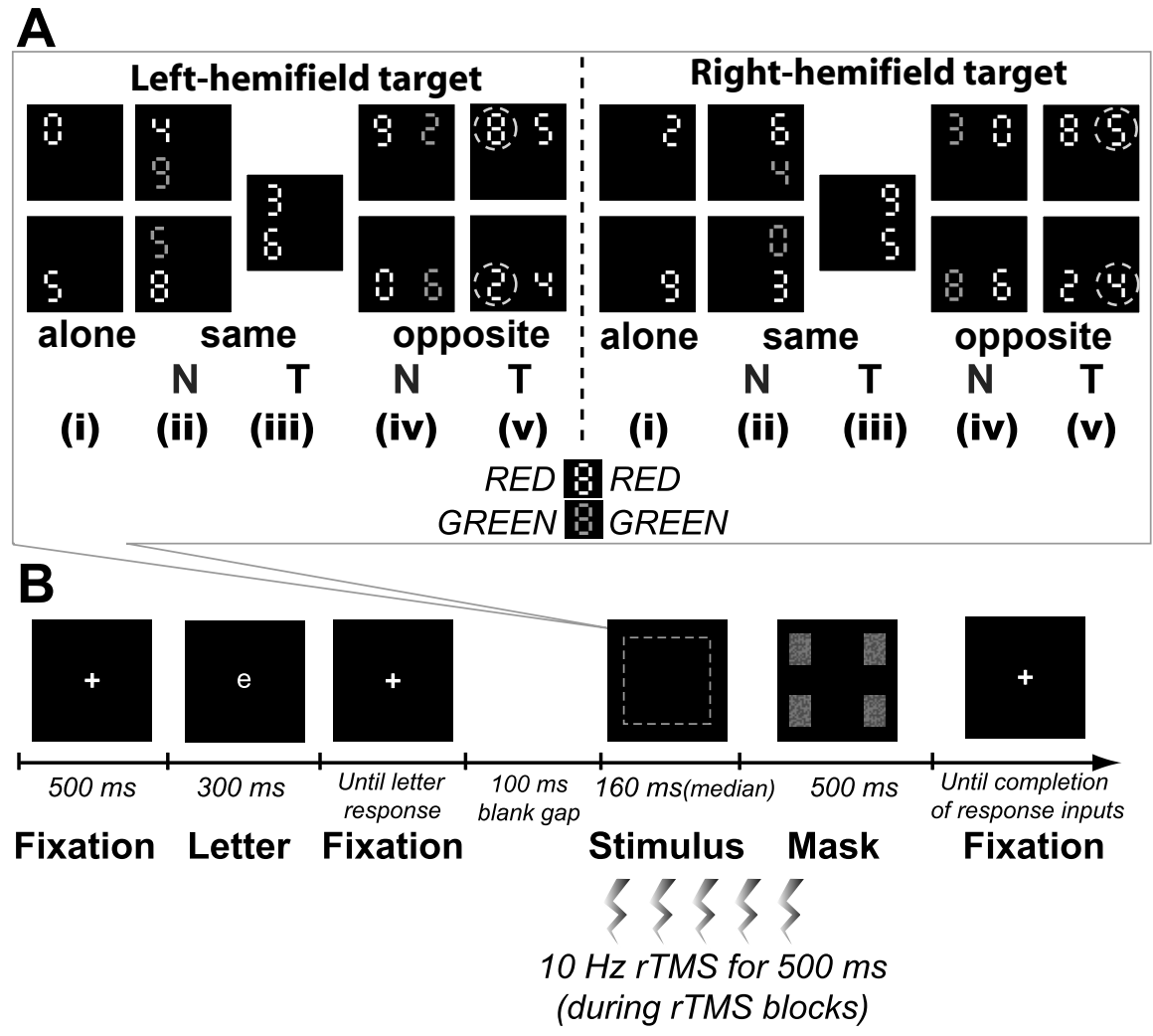

Figure 1. A, Example stimulus arrays from the partial-report experimental task, with targets assigned as red (digits in white) and nontargets as green (digits in gray) for the purposes of illustration. Each black-background square here illustrates one possible display type. These different types of display can be organized into five conditions of presentation in each hemifield: i, one target alone; ii, iii, one target accompanied with a same-hemifield nontarget (ii) or target (iii); iv, v, one target accompanied with an opposite-hemifield nontarget (iv) or target (v). Note that those arrays presenting one target in each hemifield concurrently (v) can provide separate scores for the left and right target, respectively. The dashed circles surrounding the digit in condition (v) were not present in the experiment but are added here to emphasize the scored target in a given display condition. N, Nontarget; T, target. $\boldsymbol{B}$, Temporal sequence of a single trial. Participants were required first to report a small white letter in the center to encourage central fixation (also monitored by eye tracking), which was followed by the brief stimulus array (the dashed rectangle here indicates one of the possibilities shown above, in $A$ ) and a mask. During the right or left PPC rTMS blocks, trains of rTMS pulses at $10 \mathrm{~Hz}$ for $500 \mathrm{~ms}$ (indicated schematically by lightning flashes) started synchronously with the onset of the stimulus array.

to other stimuli. A measure of top-down control is determined by the ratio of attentional weights for targets (here items with the task-relevant color) versus nontargets (with the task-irrelevant color here). Good top-down control corresponds to task-relevant items being given stronger weights.

The target-defining property in our task was nonspatial (color), whereas PPC is often assumed to contribute primarily to spatial selection (Driver and Vuilleumier, 2001). However, Tsal and Lavie $(1988,1993)$ proposed that visual selection by nonspatial properties such as color may be implemented by selecting the location of the relevant color. PPC might thus fulfill a role in selecting relevant locations even when color defines the targets (Toth and Assad, 2002). Our results showed that rTMS over right (but not left) PPC affected the efficiency of top-down selection by color in a spatially specific manner, with relative suppression of top-down control for the left hemifield but relative enhancement for the right hemifield, particularly for bilateral displays.

\section{Materials and Methods}

Participants. Nine healthy volunteers (three female, age 26-34 years) gave informed consent. All were right-handed according to the Edinburgh Inventory (Oldfield, 1971). The experimental protocol was approved by the local ethical committee and conformed to the declaration of Helsinki and safety guidelines for rTMS research (Wassermann, 1998).
Stimuli and procedure (partial-report experimental task). The general procedure was modified from a previous study (Duncan et al., 1999) (see also Peers et al., 2005) that studied effects of permanent brain damage with Bundesen's (1990) TVA approach.

Stimulus arrays comprised one or two digits $\left(\sim 0.5^{\circ}\right.$ in height $)$ at the corners of an imaginary square $\left(9.4^{\circ} \times 9.4^{\circ}\right)$ centered on fixation against a black background (Fig. 1A). Digits were randomly chosen from the set 02345689 in red [Commission Internationale de l'Eclairage (CIE), $x=0.618, y=0.346]$ and/or green (CIE, $x=0.279, y=0.577)$ matched for luminance $\left(20 \mathrm{~cd} / \mathrm{m}^{2}\right)$. At the beginning of each session, participants were instructed that either the red digits or the green digits were targets for the upcoming session. Targets were thus flexibly defined by color, in an instruction-dependent or top-down manner. Figure $1 A$ illustrates the possible presentation conditions. A target item may appear alone (four display types, corresponding to each corner of the imaginary square), accompanied by a nontarget (eight display types, because with a target at four possible positions, the nontarget could appear in the same or opposite hemifield), or accompanied by another target (four display types, corresponding to same-hemifield target columns or opposite-hemifield rows). Diagonal arrangements were not possible in the two-item displays (Fig. 1A). Each type of display was presented equally often in a random order.

As illustrated in Figure $1 B$, each trial began with a central cross $\left(0.3^{\circ}\right.$, white color $)$. To further encourage central fixation, this was replaced for $300 \mathrm{~ms}$ by a small central letter $\left(0.5^{\circ}\right.$ in height, white color) that participants immediately read aloud. The experimenter then pressed a key, and, $100 \mathrm{~ms}$ later, the stimulus array was presented briefly (median duration of $160 \mathrm{~ms}$; mean duration \pm SD across the four testing sessions for individual participants ranging from 125 to $205 \pm 14$ to $31 \mathrm{~ms}$, see below). This array was masked by a rectangular pattern with random pixels in red and green. Participants verbally reported the identity of any digits in the specified target color [e.g., reporting all red digit(s) while ignoring any green digit or vice versa] at their own speed. For displays presenting two target digits, participants had to report both in any order and to indicate their order of report. Their verbal responses were keyed in by the experimenter.

During the experiment, participants sat with head fixed by a chinrest and head strap. Stimuli were presented on a 17 -inch video graphics array display (Trinitron Multiscan 200 GS; Sony, Tokyo, Japan) at $100 \mathrm{~Hz}$ and $1024 \times 768$ pixel resolution, with a viewing distance of $57 \mathrm{~cm}$. Presentation of stimuli and response recording were controlled with E-prime 1.0 (Psychology Software Tools, Pittsburgh, PA); fixation was monitored by an infrared limbus tracking technique (IRIS; Skalar Medical, Delft, the Netherlands) and analyzed with DASYLab 5.0 (DATALOG, Mönchengladbach, Germany), which confirmed that participants maintained central fixation and that rTMS did not trigger blinking responses.

Participants performed four sessions of this task over 4 separate days, two with red as the specified target color and two with green as the target color. Each session consisted of an initial practice phase followed by four experimental blocks. During the practice phase, the duration of the stimulus array was set by a staircase procedure (Cornsweet, 1962), adjusting durations by $10 \mathrm{~ms}$ every eight trials if needed, to achieve $75 \%$ accuracy over 16 consecutive target-alone trials (averaging over the four possible target locations). The obtained duration of the stimulus array was then 
applied to all four subsequent experimental blocks, including two blocks of no rTMS, one block of left PPC rTMS, and one block of right PPC rTMS, in pseudorandom order with 10 min breaks between blocks. Given that each single session used the same display duration for all rTMS conditions (no rTMS, right or left PPC rTMS) and that the durations were functionally equivalent (i.e., all set at $75 \%$ accuracy for targetalone trials), data collected across sessions were pooled together for each individual participant. Each block consisted of 128 trials, yielding a total of 1024 trials for the no rTMS condition and 512 trials for right or left PPC rTMS conditions per participant.

Stimuli and procedure (color-report control task). To ensure that any rTMS effects on top-down selection by task-relevant color in the above partial-report task were not attributable to any effects on gross color perception, we also conducted a control task of color report (Duncan et al., 1999). The stimulus array was now a single digit in red or green at one of the four possible positions (eight display types); these positions were identical to those used in the partial-report task. Each type of display was equally probable. Participants gave verbal reports for the color of any digit presented (i.e., reporting "red" or "green") at their own speed. Participants completed this task in one session, which began with practice to determine the duration equivalent to $75 \%$ accuracy, followed by four experimental blocks in pseudorandom order. The four blocks consisted of two blocks without and two blocks with PPC rTMS (one for the left and one for the right PPC), with 64 trials in each block.

rTMS. Magnetic stimulation was generated by a Magstim Super Rapid stimulator (Magstim, Whiteland, UK) connected to a $70 \mathrm{~mm}$ figure-ofeight coil held by a mechanical arm. The sites of rTMS were specified by frameless stereotaxy, using a Polaris infrared tracking device (Northern Digital, Waterloo, Ontario, Canada) and Brainsight software (Rogue Research Inc, Montreal, Quebec, Canada). With coregistration of the $\mathrm{T}_{1}$ weighted magnetic resonance brain image to surface landmarks of the head in each participant, PPC stimulation was centered on the dorsal part of the angular gyrus between the intraparietal sulcus and the upper end of the superior temporal sulcus (for an example, see Fig. 2). Each participant's brain image was also spatially normalized to Talairach space ( $\mathrm{Ta}-$ lairach and Tournoux, 1988) using FSL software (Jenkinson and Smith, 2001, see www.fmrib.ox.ac.uk/fsl), which uses the canonical Montreal Neurological Institute (MNI) template. This gave mean coordinates of the stimulation center at $43,-65,51$ (SD of $5,6,4 \mathrm{~mm}$ ) for the right PPC and $-40,-65,53$ (SD of 3, 3, $4 \mathrm{~mm}$ ) for the left PPC.

During blocks with rTMS in the partial- and color-report tasks, rTMS was delivered from the onset of each stimulus display, in a train of $10 \mathrm{~Hz}$ for $500 \mathrm{~ms}$ (Fig. $1 B$ ) at $60 \%$ of the maximum stimulator output. The intensity/frequency of the rTMS was chosen to be well within the safety guidelines (Wassermann, 1998), whereas the timing and duration of the rTMS ensured that it should overlap with any critical processing in PPC (i.e., during and immediately after presentation of each brief visual display while participants performed the task). Such "on-line" magnetic stimulation, delivered in a small number of pulses (usually one to five) at frequencies of $\sim 10-50 \mathrm{~Hz}$, time locked to discrete trials in a task, have been used to transiently disrupt functions of the brain region targeted with TMS in many previous studies (Day et al., 1989; Pascual-Leone et al., 1994; Oliveri et al., 1999; Rushworth et al., 2001; Bjoertomt et al., 2002; Campana et al., 2002). The effects of on-line TMS, as used here, can be thought of as transiently introducing neural "noise" into the area stimulated (for review, see Walsh and Cowey, 2000; Walsh and PascualLeone, 2003). Many TMS studies adopting this interference approach have created a variety of "virtual lesions"; that is, behavioral changes mimicking those produced by lesions involving the same region [suppression of visual detection (Amassian et al., 1989), visual extinction (Pascual-Leone et al., 1994), prolongation of visual search (Ashbridge et al., 1997), akinetopsia (Walsh et al., 1998), aspects of spatial neglect (Fierro et al., 2000), or acalculia (Gobel et al., 2001)], thereby allowing tests of functional localization. Note that TMS differs from other types of neural stimulation [e.g., with implanted electrodes (Moore and Armstrong, 2003)] in many respects but can nevertheless similarly modulate a given region transiently to allow inferences about its function.

Data analysis. Performance was assessed with two types of analysis. First, we considered the raw performance scores, in an assumption-free
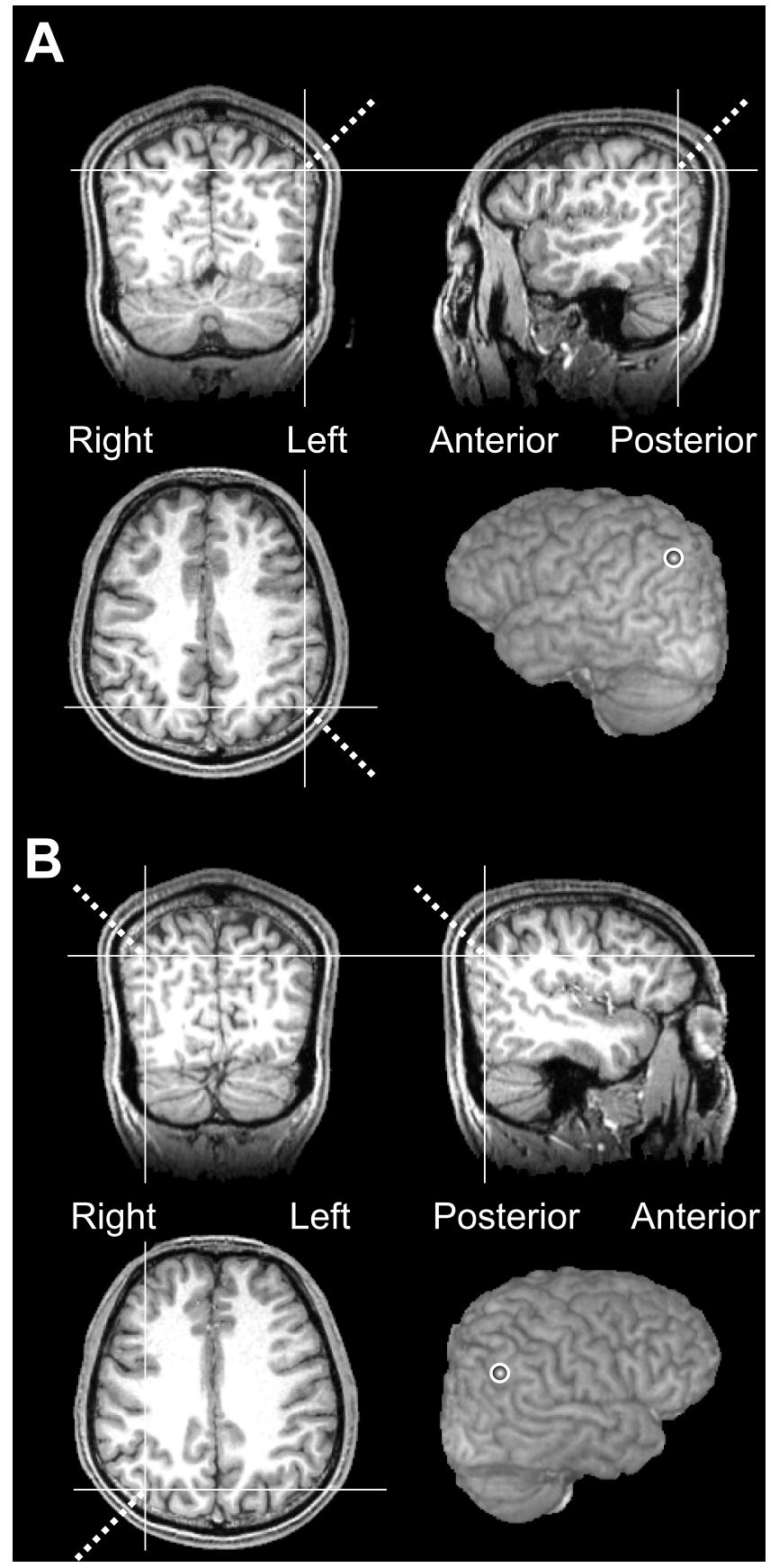

Figure 2. The stimulation centers of rTMS over the left PPC $(\boldsymbol{A})$ and the right $\operatorname{PPC}(\boldsymbol{B})$ in the $\mathrm{T}_{1}$-weighted structural image of one participant. The MNI coordinates of the stimulation center (white circle) in this participant were located at $-42,-64,50$ for the left PPC and 43, -66 , 51 for the right PPC. Dashed lines represent the projected orientation perpendicular to the surface of the coil. For mean coordinates across participants, see Materials and Methods.

approach that nevertheless still demonstrated that the general performance patterns were in accord with Bundesen's (1990) TVA approach. We then proceeded to a second analysis of the parameters as fitted by the TVA model. In both cases, effects of various conditions were assessed by repeated-measures ANOVA (i.e., each participant contributing a score for each condition) with Greenhouse-Geisser correction. Paired comparisons were performed via Newman-Keuls tests. How raw scores are transformed into TVA parameters is briefly outlined below (for additional details, justification, and discussion, see Bundesen, 1990; Duncan et al., 1999).

According to TVA, when a target is presented alone in location $i$, 
performance $P_{i}$ is closely approximated by an exponential function (Shibuya and Bundesen, 1988; Bundesen, 1998):

$$
P_{i}=1-\frac{1}{e^{A_{i}}},
$$

where $A_{i}$ is proportional to the sensory effectiveness for constant exposure duration.

When two objects are presented together, the competition between them is reflected by weight setting. Performance for a target in location $i$ accompanied by a nontarget in location $j$ is given by the following:

$$
P_{i}=1-\frac{1}{e^{A_{i} w_{i}(T) /\left(w_{i}(T)+w_{j}(N)\right)}},
$$

where $w_{i}(T)$ is the attentional weight of a target in location $i$, and $w_{j}(N)$ is the attentional weight of a nontarget in location $j$.

Performance for a target in location $i$ accompanied by a second target in location $j$ is given by the following:

$$
P_{i}=1-\frac{1}{e^{A_{i} w_{i}(T) /\left(w_{i}(T)+w_{j}(T)\right)}} .
$$

With $w(N)$ and $w(T)$ derived in each hemifield, their ratio $w(N) / w(T)$ is designated as $\alpha$ (typically $\alpha<1$ ), which indicates the efficiency of topdown control. When $\alpha$ is close to zero, only targets receive significant processing (thus indicating effective exclusion of nontargets, corresponding to effective top-down selection on the Bundesen account). When $\alpha$ approaches 1 , adding a target or a nontarget to an array has similar interference effects for the correct report of the accompanying target (thus indicating that targets and nontargets are now treated equivalently, corresponding to a lack of top-down selection).

For each participant during different rTMS conditions, a TVA trialby-trial fitting program (available at www.psy.ku.dk/cvc/tva) was used to obtain estimates of the above parameters $\left[A_{i}, w_{i}(T)\right.$, and $w_{i}(N)$ for each of the four possible stimulus locations] that gave a maximum-likelihood fit to 20 raw performance scores (four target positions under five conditions of visual presentation, i.e., presented alone, accompanied with a samehemifield nontarget/target, or accompanied with an opposite-hemifield nontarget/target) (Fig. $1 \mathrm{~A}$ ).

For both raw performance scores and the various TVA parameters derived from them, initial inspection revealed similar outcomes for the upper and lower positions in the same hemifield. Measurements for each hemifield were therefore obtained by collapsing over upper and lower positions.

\section{Results}

All participants completed the task without any adverse effects. rTMS over the PPC did not elicit motor twitches or sensory paresthesias.

\section{Partial-report experimental task}

Figure 3 shows the mean performance during left PPC rTMS, no rTMS, and right PPC rTMS conditions, for targets in the left or right hemifield, under various display conditions. Note that Figure 3 suggests a very similar pattern of performance for the left PPC rTMS and no rTMS conditions (top two panels), whereas the right PPC rTMS condition (bottom panel) indicates a different pattern of performance. This was confirmed by the analyses below, which revealed an impact of right PPC but not left PPC rTMS on performance.

Although participants were free to choose the reporting order for two-target displays, most participants reported in a left-thenright (for bilateral displays) or upper-then-lower (for unilateral displays) order, regardless of rTMS conditions. Left-then-right report order might contribute to (or reflect) the apparent trend for slightly better performance for left than right targets overall (Fig. 3), but, in fact, this did not approach significance $\left(F_{(1,8)}=\right.$
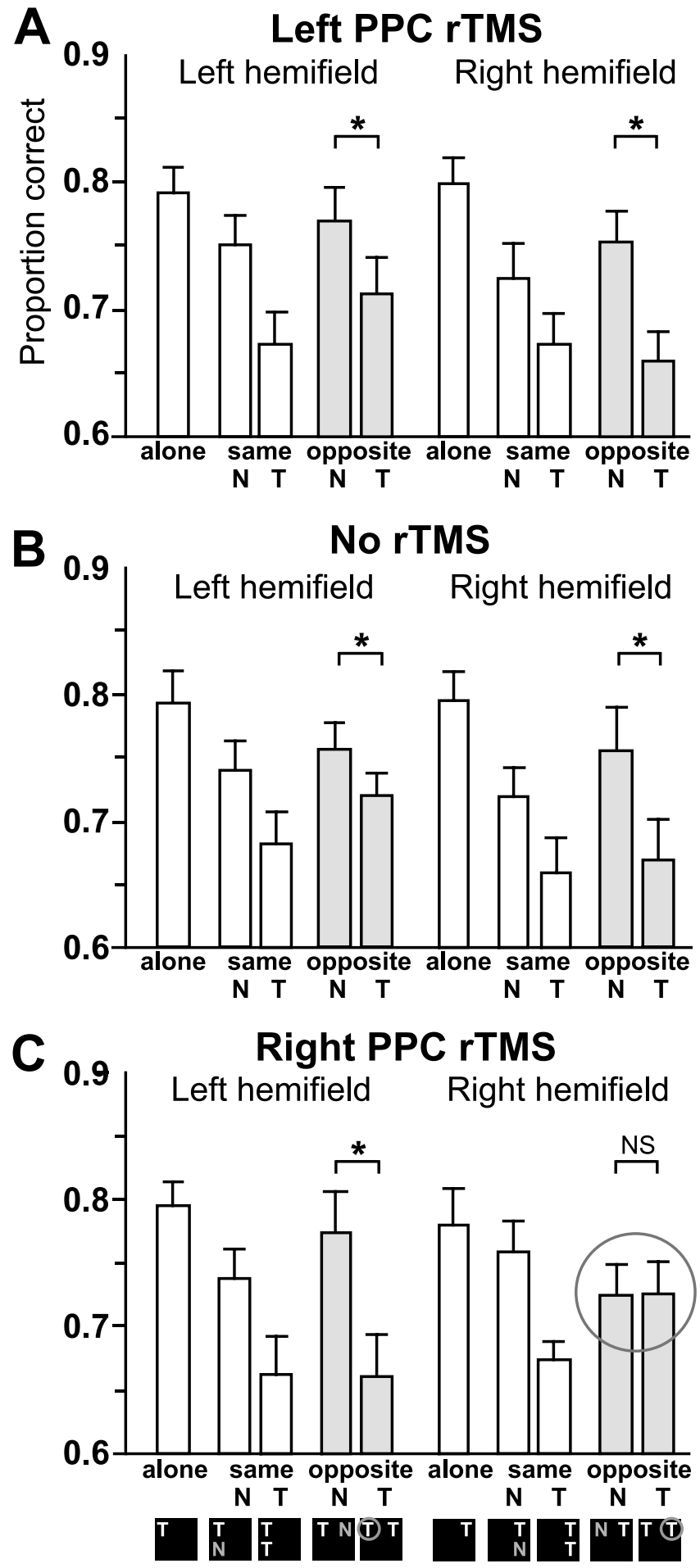

Figure 3. Mean proportion of correct report of the target for left PPC rTMS (A), no rTMS (B), and right PPC rTMS ( $($ ) in the partial-report task. Note that the pattern for right PPC rTMS (see the shaded bars, especially the rightmost circled pair) is different from those for left PPC or no rTMS (compare the shaded bars across panels). Scores are shown separately for targets in the left hemifield (leftmost 5 bars in each panel) or right hemifield. Data are further divided according to five display conditions (see the labels under each panel and the schematic displays under the bottom panel): a target alone; accompanied with a same-hemifield nontarget (N) or with a same-hemifield target (T); and accompanied with an opposite-hemifield nontarget or with an opposite-hemifield target. The schematic displays depict nontargets as $\mathrm{N}$ and targets as $\mathrm{T}$, while only showing examples for targets at the upper position (for those at the lower position, see Fig. 1A). The circle surrounding one of the Ts in the T-T bilateral displays was not present in the experiment but is added here to emphasize the scored target in a given condition. Error bars correspond to SE. The asterisked brackets $(p<0.05)$ and one bracket notifying nonsignificance (NS) draw attention to critical pairs of conditions. 
0.22, $p=0.65$ for left vs right targets in two-element displays, when collapsing over other conditions). It did not reach significance even for those conditions in which the trend was most pronounced (i.e., for target-target row displays under no rTMS or left PPC rTMS, $p>0.1$ in both cases) and hence will not be discussed further. Two participants intermittently reported two targets in the reverse order, but the frequencies of this were similarly low (3-4\%) for all rTMS conditions. In summary, rTMS did not affect the order of report, so the rTMS effects described below cannot reflect report order.

For ease of exposition, we first characterize the overall pattern regardless of rTMS and then move on to assessing any impact of rTMS, initially on raw performance scores and subsequently on the parameters calculated by the TVA approach.

\section{Raw performance scores: the overall pattern}

The overall performance pattern is consistent with the expectations of the TVA approach. Regardless of rTMS conditions, performance for target-alone displays (single item) was impaired by adding either a nontarget or a target to produce two-item displays $\left(F_{(1,8)}=48.71, p<0.0005\right.$ for performance of one- vs twoelement displays), indicating some capacity limit. Even adding a nontarget produced some performance cost, but importantly an accompanying target (i.e., another digit in the task-relevant color) was generally more disruptive than a nontarget in the taskirrelevant color $\left(F_{(1,8)}=99.11, p<0.0005\right.$ for performance in conditions with an accompanying nontarget vs another target). This indicates some degree of top-down selection (because a greater impact from targets than nontargets indicates higher attentional weights for targets on the Bundesen account). Figure 3 shows such overall patterns, with best performance for conditions with a single target alone, then those with an accompanying nontarget, and then those with a second target. This pattern was consistent, except for one situation under right PPC rTMS, as explained in the next section.

\section{Raw performance scores: the impact of rTMS}

We began by comparing performance scores under left PPC rTMS with those under no rTMS and likewise separately for performance under right PPC rTMS relative to no rTMS. We did this as an initial step instead of comparing right and left PPC rTMS first, because starting with the latter comparison might have made it unclear which site of rTMS was producing any effect. As will be seen, left PPC rTMS and no rTMS led to equivalent performance, with only right PPC rTMS producing changes in topdown control (i.e., in the relative impact of adding a target vs a nontarget). Thus, after confirming that left PPC rTMS did not differ from no rTMS, we were then able to use left PPC rTMS as a baseline for direct comparison with right PPC rTMS in subsequent analyses, allowing us to control for any nonspecific effects attributable to rTMS (e.g., possible alerting effects from the sound of the rTMS pulses).

For target-alone (single-item) displays, performance was not affected by rTMS over either hemisphere. The comparison between left PPC rTMS versus no rTMS, in a $2 \times 2$ ANOVA with rTMS (none or left PPC) and target side (left or right) as factors, revealed no main effects and no interaction (all $F$ values $\leq 0.02$, NS). A similar analysis comparing right PPC rTMS and no rTMS likewise found no significant differences (again all $F$ values $\leq 0.93$, NS). This indicates that rTMS over left or right PPC did not affect correct identification of a target digit presented alone and thus did not induce any "sensory" impairment for either hemifield.
For the critical two-item conditions, $2 \times 2 \times 2 \times 2$ repeatedmeasures ANOVAs were conducted with factors of (1) rTMS (present or absent), (2) target side (left or right), (3) display configuration (whether the accompanying item was in the same or opposite hemifield as the scored target), and (4) relevancy of the accompanying item (nontarget or target), to separately examine any effects of left PPC rTMS versus no rTMS or of right PPC rTMS versus no rTMS (for rationale, see above). The comparison of left PPC versus no rTMS revealed only main effects of relevancy $\left(F_{(1,8)}=84.39, p<0.0005\right.$, i.e., worse performance when adding a target rather than a nontarget, as expected), with no main effects or interactions involving the rTMS factor (all $F$ values $\leq 1, p \geq 0.35$ ). Importantly, these results indicate that left PPC rTMS did not impair or change performance relative to that found with no rTMS.

In contrast, the analogous ANOVA comparing right PPC rTMS versus no rTMS for two-element displays now revealed significant influences of the rTMS manipulation. In addition to the usual main effect of relevancy $\left(F_{(1,8)}=40.21, p<0.0005\right)$, there was a three-way interaction among rTMS, target side, and relevancy of accompanying item $\left(F_{(1,8)}=8.78, p=0.018\right)$, and the four-way interaction also reached significance $\left(F_{(1,8)}=32.18\right.$, $p<0.0005)$.

One likely source for these interactions is suggested by comparing Figure 3, $B$ and $C$, which reveals the change in performance pattern for right PPC rTMS. In almost all cases, performance was better for conditions with an accompanying nontarget rather than a target, yet this pattern was not seen for the scores of right-hemifield targets in the opposite-hemifield (row) configuration during right PPC rTMS (Fig. 3C, the circled rightmost two bars, as opposed to other pairs of shaded bars; note also the particularly large difference for the fourth and fifth bars in Fig. 3C). That is, with right PPC rTMS, an added left target no longer disrupted report of a right target more than an added left nontarget, yet an added right target still disrupted report of a left target more than an added right nontarget (if anything, even more so). These changes in performance pattern thus depended on rTMS $\times$ target side $\times$ display configuration $\times$ relevancy of accompanying item, corresponding to the four-way interaction.

To investigate this further, an analysis was conducted for just the two-item conditions with three factors (target side, display configuration, and relevancy of accompanying item), now separately for right PPC rTMS and no rTMS. This revealed a threeway interaction only for right PPC $\operatorname{rTMS}\left(F_{(1,8)}=23.41, p=\right.$ $0.001)$, but there was no such interaction in the absence of rTMS $\left(F_{(1,8)}=3.15, p=0.11\right)$. Additional $2 \times 2$ ANOVAs with factors of target side and relevancy of accompanying item were implemented for same- and opposite-hemifield configurations during right PPC rTMS. These demonstrated a significant target side $X$ relevancy interaction only for opposite-hemifield row displays $\left(F_{(1,8)}=28.94, p=0.001\right)$ but not for column displays $\left(F_{(1,8)}=\right.$ $0.73, p=0.42)$. This reflects the facts that, during right PPC rTMS, report of a left target was much more impeded by adding a right target rather than a right nontarget, indicating relatively good selectivity for an added right stimulus $\left(F_{(1,8)}=22.07, p=\right.$ 0.002 ) (Fig. $3 C$, fourth and fifth bars, near the asterisk), whereas for a right target, adding a left nontarget or a left target now impeded its report to virtually the same extent, i.e., the greater impact of an added left target was eliminated, indicating relatively poor selectivity for an added left stimulus $\left(F_{(1,8)}=0.12, p=\right.$ 0.74 ) (Fig. 3C, two circled rightmost bars). No such pattern was present in the absence of rTMS (Fig. $3 B$ ) or with left PPC rTMS (Fig. 3A). 

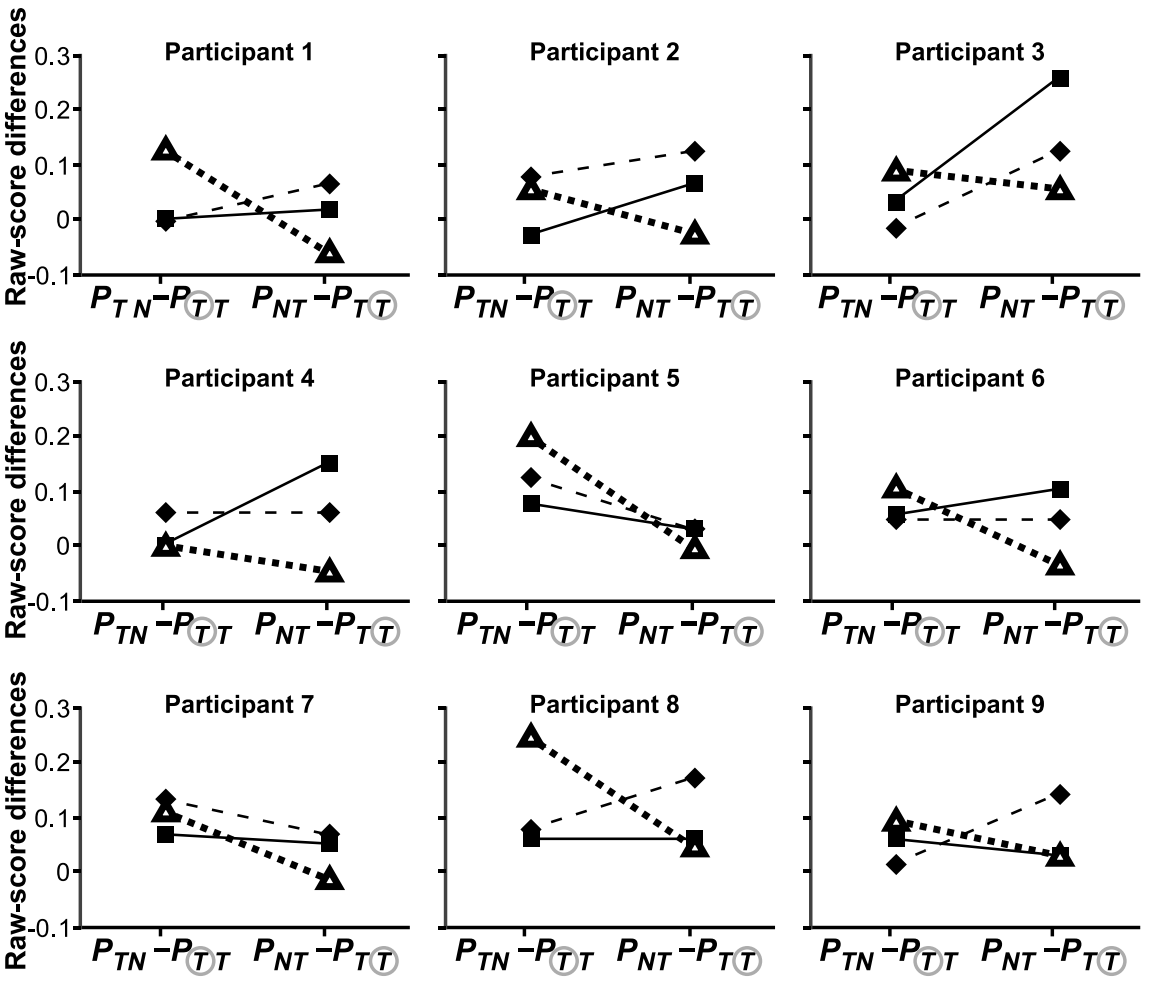

Left hemifield Right hemifield

Left hemifield Right hemifield

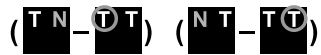

$\boldsymbol{P}_{T N}$ Performance scores for the left target with an accompanying right nontarget

$\boldsymbol{P}_{\boldsymbol{T} \text { T }}$ Performance scores for the left target with an accompanying right target

\begin{tabular}{|c|c|}
\hline & $\square-$ \\
\hline
\end{tabular}

Figure 4. The difference in raw performance scores between target-nontarget arrays and target-target arrays (i.e., $P_{T N}-P_{T T}$ for left targets and $P_{N I}-\mathrm{P}_{T T}$ for right targets) in the row displays for individual participants. Note how the dashed lines connecting triangle points (representing the data for right PPC rTMS) tend to slope down in each graph, unlike the other rTMS conditions. All participants showed a relatively lower score on the plotted performance difference for right targets (right data points in graphs) during right PPC $r T M S$, indicating relatively poorer selectivity (less differential impact of added left target than nontarget) for the left hemifield. Also, during right PPC rTMS, six of nine participants (participants 1, 3, 5, 6, 8, and 9) showed a relatively higher score on this measure for left targets (left data points in graph), indicating intact or enhanced selectivity for the right hemifield (i.e., preserved or enhanced differential impact of adding a right target vs nontarget, on report of a left target). The repeatedmeasures ANOVA statistics in Results formally confirm reliability across the group of participants.

To confirm that the above effects were consistently present in most participants (as already implied by the significant repeatedmeasures statistics), the differences in raw performance scores between target-nontarget arrays and target-target arrays (i.e., $P_{T N}-P_{T T}$ for left targets and $P_{N T}-P_{T T}$ for right targets) in the opposite-hemifield (row) conditions were plotted for each participant (Fig. 4, noting how the dashed lines connected by triangles all tend to slope down). All participants showed the critical pattern of a relatively lower score on this performance difference for report of right targets (Fig. 4, right points in each graph) during right PPC rTMS than for left PPC rTMS or none. This indicates relatively poor selectivity for the left hemifield under right PPC rTMS (i.e., less differential impact of an added target vs nontarget on the left, for report of a right target). Also, during right PPC rTMS, six of nine participants (participants 1, 3, 5, 6, 8, and 9) showed a relatively higher score on this performance difference for the report of left targets (Fig. 4, left points in each graph), suggesting some tendency for relatively improved (and certainly preserved) selectivity for the right-hemifield stimulus (i.e., intact differential impact of an added right target vs added right nontarget, because right targets still disrupted report of left targets more than right nontargets under right PPC rTMS; indeed, they tended to do so even more).

An additional approach for investigating the source of the four-way interaction above (i.e., rTMS $\times$ target side $\times$ configuration $\times$ relevancy of accompanying item) is to run three-way analyses (rTMS $\times$ target side $X$ relevancy) separately for row and column displays. These revealed a threeway (rTMS $\times$ target side $\times$ relevancy) interaction for row displays $\left(F_{(1,8)}=60.18\right.$, $p<0.0005)$ but not for column displays $\left(F_{(1,8)}=0.69, p=0.43\right)$. Additional comparisons dividing the relevancy factor revealed interactions between rTMS and target side when the accompanying item was a target $\left(F_{(1,8)}=34.36, p<0.0005\right)$ but not a nontarget $\left(F_{(1,8)}=1.49, p=0.26\right)$. This indicated that, for row displays with one target in each hemifield, right PPC rTMS (vs none) relatively worsened performance for reporting the left target $(p<0.05)$, shifting performance even farther away from that for the corresponding nontargettarget array, whereas right PPC rTMS actually benefited performance for a right target in target-target rows $(p<0.05)$, with right-target performance for these now approaching the level for the corresponding nontarget-target array. The worsened performance for the left target specifically in row target-target displays here, under right PPC rTMS, resembles the contralesional extinction (i.e., failure to report a contralesional target in the presence of an ipsilesional target) in many right parietal patients (Vuilleumier and Rafal, 2000; Driver and Vuilleumier, 2001), whereas the improvement for reporting the right target from row target-target displays may be reminiscent of improved performance for stimuli ipsilateral to the TMS in some previous work (Seyal et al., 1995; Oliveri et al., 1999; Rushworth et al., 2001), although note that the present effects did not apply for single-target conditions (see above) and may thus be more analogous to extinction.

Our final analysis on the raw performance score, before application of TVA, directly compared right PPC rTMS with left PPC rTMS. (Thus far, we have shown that right PPC rTMS differed in its effects on performance from no rTMS, whereas left PPC rTMS did not. This was an essential first step for knowing which type of rTMS was effective.) The direct comparison of right and left rTMS, which we focus on henceforth, controls for any nonspecific effects of rTMS such as alerting caused by the sound of rTMS. A repeated-measures ANOVA comparing right versus left PPC rTMS for two-item displays showed similar results to the previous comparison of right PPC versus no rTMS, including a main effect of relevancy $\left(F_{(1,8)}=148.98, p<0.0005\right)$, a three-way interaction involving rTMS condition, target side, and relevancy 
Table 1. TVA parameters in the partial-report experimental task: values of sensory effectiveness $A_{i}$, spatial weights for targets $w_{i}(T)$, and efficiency of control $\alpha$ for each individual participant

\begin{tabular}{|c|c|c|c|c|c|c|c|c|c|c|c|c|c|c|c|c|c|c|}
\hline \multirow[b]{3}{*}{ Participant } & \multicolumn{6}{|c|}{ Parameter $A_{i}$} & \multicolumn{6}{|c|}{ Parameter $w_{i}(T)$} & \multicolumn{6}{|c|}{ Parameter $\alpha$} \\
\hline & \multicolumn{2}{|c|}{ Left PPC rTMS } & \multicolumn{2}{|c|}{ No rTMS } & \multicolumn{2}{|c|}{ Right PPC rTMS } & \multicolumn{2}{|c|}{ Left PPC rTMS } & \multicolumn{2}{|c|}{ No rTMS } & \multicolumn{2}{|c|}{ Right PPC rTMS } & \multicolumn{2}{|c|}{ Left PPC rTMS } & \multicolumn{2}{|c|}{ No rTMS } & \multicolumn{2}{|c|}{ Right PPC rTMS } \\
\hline & Left & Right & Left & Right & Left & Right & Left & Right & Left & Right & Left & Right & Left & Right & Left & Right & Left & Right \\
\hline 1 & 2.41 & 1.54 & 1.88 & 1.45 & 1.87 & 1.41 & 0.81 & 0.50 & 0.98 & 0.70 & 0.80 & 0.75 & 0.45 & 0.55 & 0.63 & 0.65 & 0.61 & 0.14 \\
\hline 2 & 1.54 & 2.12 & 1.68 & 1.74 & 1.72 & 2.23 & 0.72 & 0.50 & 0.71 & 0.65 & 0.54 & 0.75 & 0.32 & 0.60 & 0.28 & 0.62 & 0.70 & 0.42 \\
\hline 3 & 1.81 & 2.25 & 1.84 & 2.00 & 1.98 & 1.77 & 0.89 & 0.54 & 0.79 & 0.45 & 0.61 & 0.88 & 0.47 & 0.70 & 0.14 & 0.40 & 0.56 & 0.38 \\
\hline 4 & 2.44 & 2.17 & 2.52 & 2.77 & 2.40 & 2.41 & 0.93 & 0.78 & 0.96 & 0.88 & 0.86 & 0.81 & 0.34 & 0.40 & 0.49 & 0.80 & 0.54 & 0.23 \\
\hline 5 & 2.31 & 2.02 & 2.03 & 1.89 & 1.92 & 1.99 & 0.87 & 0.87 & 0.90 & 0.87 & 0.71 & 0.84 & 0.44 & 0.29 & 0.29 & 0.35 & 0.21 & 0.24 \\
\hline 6 & 2.34 & 2.26 & 2.65 & 2.18 & 2.74 & 2.28 & 0.90 & 0.83 & 0.91 & 0.90 & 0.78 & 0.92 & 0.48 & 0.42 & 0.39 & 0.37 & 0.57 & 0.29 \\
\hline 7 & 1.81 & 2.04 & 1.78 & 1.99 & 2.10 & 2.38 & 0.93 & 0.91 & 0.90 & 0.85 & 0.77 & 0.89 & 0.39 & 0.52 & 0.61 & 0.45 & 0.95 & 0.45 \\
\hline 8 & 1.98 & 2.27 & 1.77 & 2.57 & 2.63 & 1.96 & 0.91 & 0.85 & 0.99 & 0.78 & 0.47 & 0.93 & 0.24 & 0.49 & 0.49 & 0.84 & 0.75 & 0.22 \\
\hline 9 & 2.09 & 2.62 & 2.57 & 2.13 & 2.22 & 2.13 & 0.67 & 0.81 & 0.69 & 0.83 & 0.59 & 0.97 & 0.30 & 0.73 & 0.48 & 0.40 & 0.60 & 0.48 \\
\hline
\end{tabular}

of accompanying item $\left(F_{(1,8)}=11.45, p=0.01\right)$, and a four-way interaction $\left(F_{(1,8)}=18.33, p=0.003\right)$ again (Fig. 3).

To summarize, the report of a target was in general more impeded by adding another target than a nontarget, indicating a degree of top-down control that gives preferential processing for targets rather than nontargets. Whereas performance under left PPC rTMS did not differ from no rTMS, right PPC rTMS did affect the raw performance scores and changed the general pattern of performance. For two-target (row) displays with one target in each hemifield, right PPC rTMS relatively decreased performance scores for the left target while increasing those for the right one, relative to no rTMS or left PPC rTMS. Under right PPC rTMS only, adding a target on the left no longer impeded the report of a right target any more than adding a left nontarget (Fig. $3 C$, two circled rightmost bars). This elimination of the difference between a left nontarget and a left target in their impact on the report of a right target suggests disruption of top-down influences for the left hemifield. Conversely, also under right PPC rTMS, adding a right target now became even more disruptive than a right nontarget in compromising report of a left target (Fig. 3C, fourth and fifth bars), suggesting well preserved topdown control within the right hemifield under right PPC rTMS. These points will now be clarified and confirmed further by analysis of the parameters derived by Bundesen's (1990) TVA approach.

\section{Analysis of TVA parameters}

Parameters of the best fits of the TVA were calculated for different rTMS conditions in each participant. Best fits accounted for $58-$ $97 \%$ (median, $88 \%$ ) of the variance in the no rTMS conditions, 64-95\% (median, 87\%) in the left PPC rTMS conditions, and 53-97\% (median, 83\%) in the right PPC rTMS conditions. The measure of each parameter for right PPC rTMS will be compared first with that for left PPC rTMS given that this controls for nonspecific effects of rTMS (then with that for no rTMS, for completeness). Parameters from the best fits of the TVA to each participant's individual data are shown in Table 1.

Sensory effectiveness, $A_{i}$, was evaluated by the index $A_{L} /\left(A_{L}+\right.$ $A_{R}$ ) to reflect the relative difference across hemifields (an index larger or smaller than 0.5 means a leftward or rightward bias, respectively) (for its distribution, see Fig. 5A). There was neither a significant difference for right versus left PPC rTMS $\left(F_{(1,8)}=\right.$ $1.31, p=0.29)$ nor for right PPC versus no rTMS $\left(F_{(1,8)}=0.35\right.$, $p=0.57)$, as expected from their similar raw performance scores for target-alone trials. This indicates that rTMS over the left or right PPC did not affect the basic sensory processing of a single target in either hemifield.

The spatial bias in overall weighting (regardless of target/nontarget) was evaluated by the index $w_{L} /\left(w_{L}+w_{R}\right)$ (for its distribution, see Fig. $5 B$ ), where $w_{L}$ was calculated as the mean of $w_{L}(N)$ and $w_{L}(T)$ and analogously for $w_{R}$. This index was again similar for right versus left PPC rTMS $\left(F_{(1,8)}=3.16, p=0.11\right)$ and for right PPC versus no rTMS $\left(F_{(1,8)}=2.32, p=0.17\right)$, meaning that rTMS over the left or right PPC did not change the overall spatial weights (that collapse weights for targets and nontargets together).

The critical parameter representing the efficiency of top-down control, $\alpha$, is indexed as $w(N) / w(T)$ for each hemifield (in effect, the extent of impairment in reporting a target by adding another target instead of a nontarget). Although this parameter is quite variable between individuals (Duncan et al., 1999), right PPC rTMS systemically shifted $\alpha_{L}$ toward higher values (i.e., lower efficiency of top-down control for the left hemifield) while shifting $\alpha_{R}$ toward lower values (i.e., higher efficiency of top-down control for the right hemifield) (Fig. 5C). A $2 \times 2$ repeatedmeasures ANOVA with rTMS (left or right PPC) and hemifield (left or right) as factors revealed a significant interaction $\left(F_{(1,8)}=\right.$ 20.93, $p=0.002$ ) (Fig. 6). This interaction reflected right PPC rTMS increasing $\alpha_{L}$ and decreasing $\alpha_{R}$ compared with the same parameters during left PPC rTMS (all $p$ values $<0.05$ ). Moreover, $\alpha_{R}$ was significantly lower than $\alpha_{L}$ during right PPC rTMS ( $p<$ 0.05 ) but not during left PPC rTMS (NS). The same pattern was observed when comparing right PPC with no rTMS instead $\left(F_{(1,8)}=19.56, p=0.002\right.$ for the two-way interaction $)$. Thus, the efficiency of top-down control, while being similar across space with no rTMS and also with left PPC rTMS, was differentially impaired for the left hemifield but enhanced for the right hemifield by right PPC rTMS.

\section{Summary of partial-report results}

Performance on the partial (i.e., selective) report task generally showed a greater disruptive effect of an added target than a nontarget, suggesting the presence of some top-down control. Right PPC rTMS modulated this performance pattern, such that adding a left target no longer impeded report of a right target any more than adding a left nontarget, although adding a right target still impaired report of a left target more strongly than adding a right nontarget (indeed even more so). Formal TVA analysis confirmed that the efficiency of top-down control was altered by 

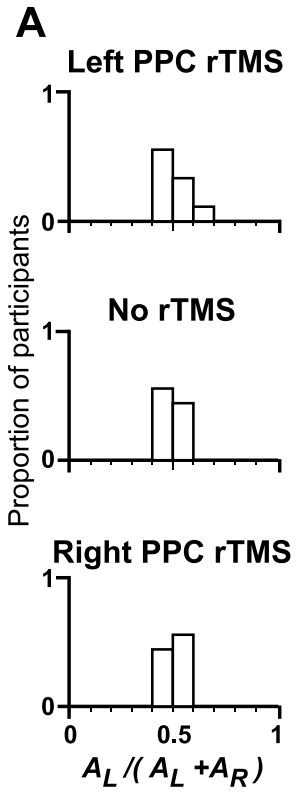

B

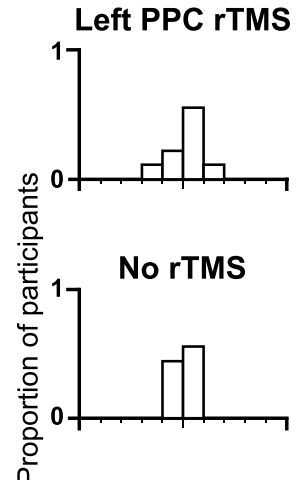

Right PPC rTMS

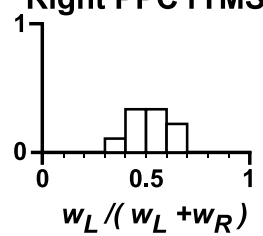

C
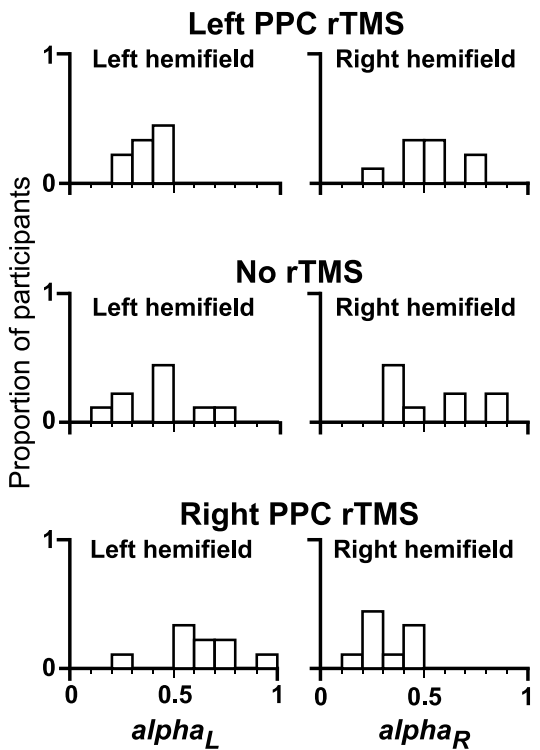

Figure 5. Distribution histograms of TVA parameters, as proportions of participants for the following: $A$, the sensory effectiveness parameter, namely $A_{L} /\left[A_{L}+A_{R}\right] ; \boldsymbol{B}$, the spatial bias regardless of target/nontarget, namely $w_{L} /\left[w_{L}+w_{R}\right] ;$ and $\boldsymbol{C}$, the efficiency of top-down control for each hemifield, $\alpha_{L}$ and $\alpha_{R}$. The distribution for each parameter is shown separately for conditions of left PPC rTMS (top panel in each case), no rTMS (middle panel in each case), and right PPC rTMS (bottom panel in each case). During right PPC rTMS, the distribution of $\alpha$, was shifted toward relatively higher values (decreased efficiency of top-down control), whereas the distribution of $\alpha_{R}$ was shifted toward relatively lower values (increased efficiency of top-down control) compared with left PPC rTMS or no rTMS.

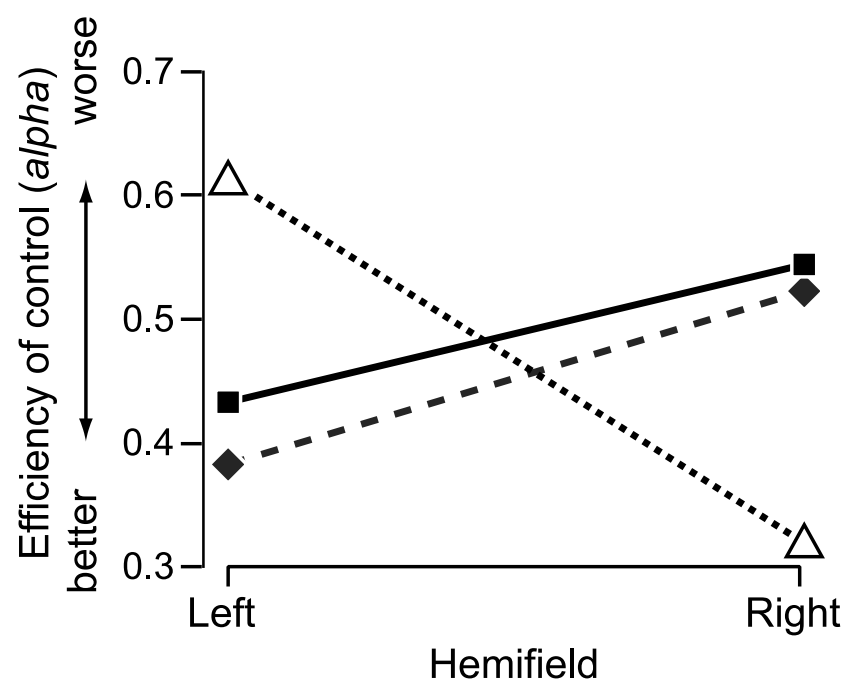

\section{$\begin{array}{ccc}\bullet--- & \square- & \triangle \cdots \cdots \\ \text { Left PPC rTMS } & \text { No rTMS } & \text { Right PPC rTMS }\end{array}$}

Figure 6. Mean estimates of $\alpha$ in each hemifield. Right PPC rTMS relatively increased the $\alpha$ value for the left hemifield while decreasing the $\alpha$ value for the right hemifield.

right PPC rTMS, being reduced for the left hemifield and (perhaps because of this) relatively enhanced for the right hemifield.

\section{Color-report control task}

A repeated-measures ANOVA with rTMS (left or right PPC) and target side as factors on the color-report data showed neither main effects nor interactions (all $p$ values $\geq 0.28$ ). Analogous

comparison of right PPC versus no rTMS also found no difference (all $p$ values $\geq 0.32$ ). For TVA analysis, performance was transformed into the estimate of $A_{i}$ (color) using Equation 1:

$$
P_{i}(\text { color })=1-\frac{1}{e^{A_{i}(\text { color })}} .
$$

For the index $A_{L}$ (color) $/\left[A_{L}\right.$ (color) $+A_{R^{-}}$ (color)], reflecting spatial bias in color discriminability, there was not a significant difference for right versus left PPC rTMS $\left(F_{(1,8)}=3.54, \mathrm{NS}\right)$ or for right PPC versus no rTMS $\left(F_{(1,8)}=1.01, \mathrm{NS}\right)$. This confirms that rTMS over either left or right PPC did not affect discrimination ability for colors, and hence the influences shown on the partial-report task above are not attributable to indirect effects on gross color perception.

\section{Discussion}

We applied rTMS to right or left posterior parietal cortex, or gave none, during a partial-report (i.e., selective-report) task in which participants reported digits in a specified target color while trying to ignore nontarget digits in another color from a brief masked display. We found that $10 \mathrm{~Hz}$ rTMS over right PPC (but not left PPC), in a time period overlapping with the stimulus and extending into its subsequent processing, altered top-down visual selection (as assessed by the differential impact of added targets vs nontargets on performance) across space. rTMS to right PPC produced a relative loss of topdown selection in the left hemifield but relative enhancement of this in the right hemifield. These effects were particularly pronounced for row displays, which had competing stimuli in both hemifields concurrently.

These effects of right PPC rTMS were not merely attributable to the presence/absence of rTMS, because they were specific to right but not left PPC rTMS. Moreover, instead of causing global, nonspecific effects on performance, right PPC rTMS had a highly condition-specific impact depending on the target side, the row/ column format, and the relevancy of an additional item in twoitem displays. Specifically, during right PPC rTMS, adding a left target no longer disrupted report of a right target any more than adding a left nontarget (Fig. 3), indicating impaired selectivity on the left side, whereas an added right target now became even more disruptive than a right nontarget in compromising report of a concurrent left target (Fig. 3), indicating improved visual selectivity on the right, perhaps as a consequence of this being impaired on the left. These effects on raw performance were echoed in TVA analyses, which indicated that right PPC rTMS influenced just the efficiency of top-down control in a spatially specific manner (Figs. 5, 6) while producing no effect on sensory effectiveness for the processing of single items, overall spatial biases, or gross color perception.

Effects of rTMS versus those of large brain lesions

TVA analyses of partial (i.e., selective) report have previously proved useful to study normal performance (Shibuya and Bundesen, 1988; Shibuya, 1993) and to investigate deficits in pa- 
tients with extensive brain damage (Duncan et al., 1999; Peers et al., 2005). Duncan et al. (1999) found some lowering of sensory effectiveness $\left(A_{i}\right)$ and of overall weighting $\left(w_{i}\right)$ regardless of target/nontarget status for the left hemifield in patients with extensive damage over the right parietal/perisylvian regions. However, such a pattern was not apparent for focal right PPC rTMS applied to normals in our study. This different outcome may reflect the broader extent of lesions in those patients (or some long-term changes that might have occurred) as opposed to the transient rTMS-induced disruption targeting at a specific region in normals here. Duncan et al. (1999) observed no specific lesion correlate of top-down control $(\alpha)$ in their patients, but this topdown parameter was highly variable even among their healthy controls (Bundesen et al., 1985; Shibuya, 1993), which could have made any distinction hard to detect. Peers et al. (2005) recently observed some deficits in top-down control in frontal and parietal patients, again using a partial-report task, but the only clear anatomical correlation for this was with lesion size. Given that top-down parameters can vary substantially between individuals (Shibuya, 1993; Duncan et al., 1999; Peers et al., 2005) (Table 1), the within-subject design that was permitted by the transient rTMS intervention here may provide a more sensitive measure for any effects on this parameter than the between-group comparisons that are typically necessary in patient studies.

\section{Spatial specificity of the right PPC rTMS effect}

The effect of right PPC rTMS on raw performance scores, and on TVA top-down parameters, was spatially specific in two respects. First, it differentially affected the left and right hemifields, with top-down selectivity being impaired on the left but improved on the right. Second, the effect was more pronounced for row displays (i.e., one competing item in each hemifield concurrently) than for column displays. This latter aspect is reminiscent of extinction commonly associated with right parietal damage (for review, see Driver and Vuilleumier, 2001; Driver et al., 2004). Typically, extinction manifests as a relative disadvantage for the contralesional (left) side and a concurrent advantage for the ipsilesional (right) side during double simultaneous stimulation from both sides. A somewhat analogous pattern (i.e., relative impairment on the left and relative enhancement on the right) was found here with bilateral (row) displays during right PPC rTMS but now specifically for top-down control parameters.

Previous TMS studies have found that disruptions in one hemisphere can lead to benefits in the other (Seyal et al., 1995; Oliveri et al., 1999; Rushworth et al., 2001), a pattern typically attributed to inter-hemispheric competition or disinhibition. The present results accord generally with those findings, showing that rTMS to right parietal cortex can have disruptive influences for the left hemifield, which may then lead to (otherwise paradoxical) relative enhancements for the right hemifield. However, they now show this pattern specifically for top-down parameters, which the TVA approach allowed us to assess for the first time here.

\section{Spatially specific role for right PPC even during color-based selection}

Targets and nontargets were distinguished only by color in the present partial-report paradigm, yet spatially specific effects (see above) of right PPC rTMS during top-down selection by color were nevertheless obtained. This provides a new line of evidence in support of previous proposals that selection of targets defined in a top-down manner by a nonspatial property (e.g., color) may be implemented by selecting the location of any item with the target-defining property (Tsal and Lavie, 1988, 1993). Interac- tions between spatial and nonspatial aspects of visual selection have now been observed in several neurophysiological studies (Treue and Martinez Trujillo, 1999; McAdams and Maunsell, 2000; Saenz et al., 2002). Event-related potentials and magnetic field recordings in humans further show that targets defined by nonspatial feature-based selection can subsequently lead to selection of the location of a likely target (Hopf et al., 2004). Moreover, in a recent study on monkeys, Toth and Assad (2002) recorded single-unit activities from the lateral intraparietal sulcus (area LIP), one possible homolog of the human PPC, while the monkeys selected an eye-movement based on the color or location of a visual cue. A number of LIP neurons were found to show colorselective responses when color became the task-relevant property for defining spatial selection, as here. Thus, there are several precedents for top-down selection by nonspatial properties leading to spatially specific selection (Quintana and Fuster, 1992, 1999). The present study directly establishes a role of the right human $\mathrm{PPC}$ in this.

\section{Hemispheric asymmetry and questions for future research}

The present results establish a key role for the right (but not left) human PPC in top-down visual selection. Such apparent hemispheric asymmetry is commonly found in human studies of attentional control (unlike more symmetric patterns in nonhuman primates), with lesion evidence (Sterzi et al., 1993; Vallar, 2001) and neuroimaging evidence (Vallar et al., 1999; Fink et al., 2001) from humans both suggesting some form of right-hemisphere specialization for spatial selection. The exact reasons for such asymmetry in the human brain remain an enduring issue, with suggestions including left-hemisphere specialization for language, as well as possible right-hemisphere specialization for representing both sides of space rather than just contralateral space (Heilman and Van Den Abell, 1980; Mesulam, 1999; Driver et al., 2004). On the latter point, it may be noteworthy that here right PPC rTMS did affect both sides of space, albeit in opposite ways (disrupting top-down selectivity on the left but enhancing it on the right, in bilateral row displays). In any case, the present evidence goes beyond previous clinical and neuroimaging observations by showing directly that transient disruption of right PPC can affect the efficiency of just top-down control (but not other TVA parameters) in a spatially specific manner. In contrast, left PPC rTMS had no such effect. Indeed, here we did not find a site in which rTMS disrupted top-down control for right hemifield (left PPC rTMS had no effect on this, whereas right PPC rTMS enhanced it).

Future work could apply the present combination of techniques (i.e., TVA analysis of partial report with on-line rTMS applied to specific sites) to determine the roles of other brain regions in each of the TVA parameters. Whereas rTMS to right PPC did not provide a complete "knock-out" of top-down control (but rather a spatial modulation of this, with disruption for the left hemifield and enhancement for the right hemifield), stimulation of more frontal regions [e.g., frontal eye fields (Grosbras and Paus, 2002; Muggleton et al., 2003; O'Shea et al., 2004; Schall, 2004)] might conceivably do so.

\section{Conclusion}

The present study combined rTMS with TVA analysis in a partialreport paradigm. The results directly confirm a role for right PPC in spatially specific aspects of top-down visual selection, as assessed by the TVA approach, even when the targets to be selected are defined by a nonspatial property such as color. 


\section{References}

Amassian VE, Cracco RQ, Maccabee PJ, Cracco JB, Rudell A, Eberle L (1989) Suppression of visual perception by magnetic coil stimulation of human occipital cortex. Electroencephalogr Clin Neurophysiol 74:458-462.

Ashbridge E, Walsh V, Cowey A (1997) Temporal aspects of visual search studied by transcranial magnetic stimulation. Neuropsychologia 35:1121-1131.

Bjoertomt O, Cowey A, Walsh V (2002) Spatial neglect in near and far space investigated by repetitive transcranial magnetic stimulation. Brain 125:2012-2022.

Broadbent DE (1958) Perception and communication. Oxford: Oxford UP.

Bundesen C (1990) A theory of visual attention. Psychol Rev 97:523-547.

Bundesen C (1998) A computational theory of visual attention. Philos Trans R Soc Lond B Biol Sci 353:1271-1281.

Bundesen C, Shibuya H, Larsen A (1985) Visual selection from multielement displays: a model for partial report. In: Attention and performance XI (Posner MI, Marin OSM, eds), pp 631-649. Hillsdale, NJ: Erlbaum.

Campana G, Cowey A, Walsh V (2002) Priming of motion direction and area V5/MT: a test of perceptual memory. Cereb Cortex 12:663-669.

Cornsweet T-N (1962) The staircase method in psychophysics. Am J Psychol 75:485-491.

Day BL, Rothwell JC, Thompson PD, Maertens de Noordhout A, Nakashima K, Shannon K, Marsden CD (1989) Delay in the execution of voluntary movement by electrical or magnetic brain stimulation in intact man. Evidence for the storage of motor programs in the brain. Brain 112:649-663.

Desimone R (1998) Visual attention mediated by biased competition in extrastriate visual cortex. Philos Trans R Soc Lond B Biol Sci 353:1245-1255.

Desimone R, Duncan J (1995) Neural mechanisms of selective visual attention. Annu Rev Neurosci 18:193-222.

Driver J, Frackowiak RS (2001) Neurobiological measures of human selective attention. Neuropsychologia 39:1257-1262.

Driver J, Vuilleumier P (2001) Perceptual awareness and its loss in unilateral neglect and extinction. Cognition 79:39-88.

Driver J, Vuilleumier P, Husain M (2004) Spatial neglect and extinction. In: The cognitive neurosciences III, Ed 3 (Gazzaniga MS, ed), pp 589-606. Cambridge, MA: MIT.

Duncan J, Bundesen C, Olson A, Humphreys G, Chavda S, Shibuya H (1999) Systematic analysis of deficits in visual attention. J Exp Psychol Gen 128:450-478.

Fierro B, Brighina F, Oliveri M, Piazza A, La Bua V, Buffa D, Bisiach E (2000) Contralateral neglect induced by right posterior parietal rTMS in healthy subjects. NeuroReport 11:1519-1521.

Fink GR, Driver J, Rorden C, Baldeweg T, Dolan RJ (2000) Neural consequences of competing stimuli in both visual hemifields: a physiological basis for visual extinction. Ann Neurol 47:440-446.

Fink GR, Marshall JC, Weiss PH, Zilles K (2001) The neural basis of vertical and horizontal line bisection judgments: an fMRI study of normal volunteers. NeuroImage 14:S59-S67.

Gobel S, Walsh V, Rushworth MF (2001) The mental number line and the human angular gyrus. NeuroImage 14:1278-1289.

Grosbras MH, Paus T (2002) Transcranial magnetic stimulation of the human frontal eye field: effects on visual perception and attention. J Cogn Neurosci 14:1109-1120.

Harter MR, Aine CJ (1984) Brain mechanisms of visual selective attention. In: Varieties of attention (Parasuraman R, Davies DR, eds), pp 293-321. Orlando, FL: Academic.

Heilman KM, Valenstein E (1979) Mechanisms underlying hemispatial neglect. Ann Neurol 5:166-170.

Heilman KM, Van Den Abell T (1980) Right hemisphere dominance for attention: the mechanism underlying hemispheric asymmetries of inattention (neglect). Neurology 30:327-330.

Hopf JM, Boelmans K, Schoenfeld MA, Luck SJ, Heinze HJ (2004) Attention to features precedes attention to locations in visual search: evidence from electromagnetic brain responses in humans. J Neurosci 24:1822-1832.

Hopfinger JB, Buonocore MH, Mangun GR (2000) The neural mechanisms of top-down attentional control. Nat Neurosci 3:284-291.
Jenkinson M, Smith S (2001) A global optimisation method for robust affine registration of brain images. Med Image Anal 5:143-156.

Kastner S, Ungerleider LG (2000) Mechanisms of visual attention in the human cortex. Annu Rev Neurosci 23:315-341.

Kastner S, Ungerleider LG (2001) The neural basis of biased competition in human visual cortex. Neuropsychologia 39:1263-1276.

Kastner S, De Weerd P, Desimone R, Ungleider LG (1998) Mechanisms of directed attention in the human extrastriate cortex as revealed by functional MRI. Science 282:108-111.

Kwong KK, Belliveau JW, Chesler DA, Goldberg IE, Weisskoff RM, Poncelet BP, Kennedy DN, Hoppel BE, Cohen MS, Turner R, Cheng H-M, Brady TJ, Rosen BR (1992) Dynamic magnetic resonance imaging of human brain activity during primary sensory stimulation. Proc Natl Acad Sci USA 89:5675-5679.

McAdams CJ, Maunsell JH (2000) Attention to both space and feature modulates neuronal responses in macaque area V4. J Neurophysiol 83:1751-1755.

Mesulam MM (1981) A cortical network for directed attention and unilateral neglect. Ann Neurol 10:309-325.

Mesulam MM (1999) Spatial attention and neglect: parietal, frontal and cingulate contributions to the mental representation and attentional targeting of salient extrapersonal events. Philos Trans R Soc Lond B Biol Sci 354:1325-1346.

Moore T, Armstrong KM (2003) Selective gating of visual signals by microstimulation of frontal cortex. Nature 421:370-373.

Muggleton NG, Juan CH, Cowey A, Walsh V (2003) Human frontal eye fields and visual search. J Neurophysiol 89:3340-3343.

Oldfield RC (1971) The assessment and analysis of handedness: the Edinburgh inventory. Neuropsychologia 9:97-113.

Oliveri M, Rossini PM, Traversa R, Cicinelli P, Filippi MM, Pasqualetti P, Tomaiuolo F, Caltagirone C (1999) Left frontal transcranial magnetic stimulation reduces contralesional extinction in patients with unilateral right brain damage. Brain 122:1731-1739.

O'Shea J, Muggleton NG, Cowey A, Walsh V (2004) Timing of target discrimination in human frontal eye fields. J Cogn Neurosci 16:1060-1067.

Pascual-Leone A, Gomez-Tortosa E, Grafman J, Alway D, Nichelli P, Hallett M (1994) Induction of visual extinction by rapid-rate transcranial magnetic stimulation of parietal lobe. Neurology 44:494-498.

Peers PV, Ludwig CJ, Rorden C, Cusack R, Bonfiglioli C, Bundesen C, Driver J, Antoun N, Duncan J (2005) Attentional functions of parietal and frontal cortex. Cereb Cortex 15:1469-1484.

Quintana J, Fuster JM (1992) Mnemonic and predictive functions of cortical neurons in a memory task. NeuroReport 3:721-724.

Quintana J, Fuster JM (1999) From perception to action: temporal integrative functions of prefrontal and parietal neurons. Cereb Cortex 9:213-221.

Reynolds JH, Chelazzi L, Desimone R (1999) Competitive mechanisms subserve attention in macaque areas V2 and V4. J Neurosci 19:1736-1753.

Rushworth MF, Ellison A, Walsh V (2001) Complementary localization and lateralization of orienting and motor attention. Nat Neurosci 4:656-661.

Saenz M, Buracas GT, Boynton GM (2002) Global effects of feature-based attention in human visual cortex. Nat Neurosci 5:631-632.

Schall JD (2004) On the role of frontal eye field in guiding attention and saccades. Vision Res 44:1453-1467.

Seyal M, Ro T, Rafal R (1995) Increased sensitivity to ipsilateral cutaneous stimuli following transcranial magnetic stimulation of the parietal lobe. Ann Neurol 38:264-267.

Shibuya H (1993) Efficiency of visual selection in duplex and conjunction conditions in partial report. Percept Psychophys 54:716-732.

Shibuya H, Bundesen C (1988) Visual selection from multielement displays: measuring and modeling effects of exposure duration. J Exp Psychol Hum Percept Perform 14:591-600.

Sperling G (1960) The information available in brief visual presentations. Psychol Monogr 74:1-29.

Sterzi R, Bottini G, Celani MG, Righetti E, Lamassa M, Ricci S, Vallar G (1993) Hemianopia, hemianaesthesia, and hemiplegia after right and left hemisphere damage. A hemispheric difference. J Neurol Neurosurg Psychiatry $56: 308-310$.

Talairach J, Tournoux B (1988) Co-planar stereotaxic atlas of the human brain. Stuttgart, Germany: Thieme. 
Toth LJ, Assad JA (2002) Dynamic coding of behaviourally relevant stimuli in parietal cortex. Nature 415:165-168.

Treue S, Martinez Trujillo JC (1999) Feature-based attention influences motion processing gain in macaque visual cortex. Nature 399:575-579.

Tsal Y, Lavie N (1988) Attending to color and shape: the special role of location in selective visual processing. Percept Psychophys 44:15-21.

Tsal Y, Lavie N (1993) Location dominance in attending to color and shape. J Exp Psychol Hum Percept Perform 19:131-139.

Vallar G (2001) Extrapersonal visual unilateral spatial neglect and its neuroanatomy. NeuroImage 14:S52-S58.

Vallar G, Lobel E, Galati G, Berthoz A, Pizzamiglio L, Le Bihan D (1999) A fronto-parietal system for computing the egocentric spatial frame of reference in humans. Exp Brain Res 124:281-286.

von Wright JM (1968) Selection in visual immediate memory. Q J Exp Psychol 20:62-68.

Vuilleumier PO, Rafal RD (2000) A systematic study of visual extinction.
Between- and within-field deficits of attention in hemispatial neglect. Brain 123:1263-1279.

Walsh V, Cowey A (2000) Transcranial magnetic stimulation and cognitive neuroscience. Nat Rev Neurosci 1:73-79.

Walsh V, Pascual-Leone A (2003) Transcranial magnetic stimulation: a neurochronometrics of mind. Cambridge, MA: MIT.

Walsh V, Ellison A, Battelli L, Cowey A (1998) Task-specific impairments and enhancements induced by magnetic stimulation of human visual area V5. Proc R Soc Lond B Biol Sci 265:537-543.

Wassermann EM (1998) Risk and safety of repetitive transcranial magnetic stimulation: report and suggested guidelines from the International Workshop on the Safety of Repetitive Transcranial Magnetic Stimulation, June 5-7, 1996. Electroencephalogr Clin Neurophysiol 108:1-16.

Yantis S, Schwarzbach J, Serences JT, Carlson RL, Steinmetz MA, Pekar JJ, Courtney SM (2002) Transient neural activity in human parietal cortex during spatial attention shifts. Nat Neurosci 5:995-1002. 